\title{
Bankruptcy Prediction Using Multilayer Perceptron Neural Networks In Jordan
}

\author{
Yusuf Ali Khalaf Al-Hroot, PhD
}

Philadelphia University, Jordan

\section{doi: 10.19044/esj.2016.v12n4p425 URL:http://dx.doi.org/10.19044/esj.2016.v12n4p425}

\begin{abstract}
This study attempts to develop bankruptcy prediction model for the Jordanian industrial sector with a recent approach-neural networks. The multilayer perceptron neural network (MPNN) approach was used to develop the bankruptcy prediction model for the Jordanian industrial companies for the period from 2000 to 2015. The samples have been divided into two subsets: the first set for developing or building the model, made up of 14 companies, of which 7 are bankrupt and 7 are non-bankrupt; while the second is a hold-out sample for testing the model, made up of 18 companies, of which 9 are bankrupt and 9 are non-bankrupt. The main variables in predicting bankruptcy were ten financial ratios. The results show that the accuracy rate of final prediction model is found to be 100 percent. While the hold-out sample testing provides that the model correctly predicted all 18 test cases.
\end{abstract}

Keywords: Bankruptcy, Financial ratios, Neural network, MPNN, Jordan

\section{Introduction:}

There are accruing attentions of bankruptcy models in the world, sometimes researchers build a model that can predicts bankruptcy before it occurs at least one year, otherwise through applying model developed in other countries such as Altman's model applied in Iran (Karamzadeh, 2013) another study of Alkhatib \& Al Bzour (2011) used the same model but applied in Jordan, and sometimes the researchers developed several models to compare them and see which model ability to predict more than others such as the study of Goss and Ramchandani, (1995). According to most of the results of studies in bankruptcy, the predictive ability differ from one country to another, and using different statistical tools to develop model give different results in predicting bankruptcy, The commonly classification models used in predicting bankruptcy studies are discriminant analysis, 
logistic regression and neural networks. Beside, Neural networks (NN) have shown high predictive ability in several studies worldwide.

\section{Research Objective}

The objective of this study is developing bankruptcy prediction model for the Jordanian industrial sector as a statistical tool for bankruptcy prediction in Jordan. Because of this position this study will develops and apply neural network statistical model for bankruptcy prediction in Jordan.

\section{Literature review and Developing Hypotheses}

The bankruptcy prediction history started since 1932. From that date predicts bankruptcy has been a subject of methodical analysis since 1932, the study of Fitzpatrick (1932) was recorded as the first study in this area.

In 1967, William Beaver used t-tests to evaluate the importance of accounting ratios within a similar pair-matched sample. The study of Altman (1968) is the common study in this field; Altman applied a new way by using multiple discriminant analysis among pair-matched samples. Another study of Ohlson (1980) used the logit regression statistic in a larger size of sample that did not include pair-matching.

The first research on bankruptcy prediction using neural networks was the study of Odom and Sharda (1990) the researchers developed a neural network (NN) model to predict bankruptcy, in this study a comparison of two methods presented; the (NN) and the discriminant analysis method to assess the predictive abilities of both methods. The results show that a (NN) has the good prediction ability. Another study of Koh and Tan (1999) the researchers developed a (NN) model to predict a firm's going concern status from six financial ratios, using data for 165 matched pairs of firms. The NN model correctly predicted all tested cases. The study suggests that NN can be a promising avenue of research and application in the going concern area.

In Jordan, the study of Alkhatib and Al Bzour (2011) they apply the models of Altman and Kida on a sample included both non-financial service and industrial companies for the period among 1990 and 2006. From the two models Altman's model outperform in company bankruptcy prediction with a 93.8\%, while Kida's model bankruptcy prediction is 69\%. The researchers conclude that Altman's model outperform Kida's model. Another study of Gharaibeh et al. (2013) this study also applied Altman and Kida models on a sample included 38 companies divided into two equal groups; Failed firms and Healthy firms during (2005-1012) in the Jordanian manufacturing firms, from the two models Altman's model outperform in company bankruptcy prediction with a $89.5 \%$ for one year before bankruptcy,65.8\% for two years before bankruptcy, and 52.6\% for three years before bankruptcy, while Kida's model bankruptcy prediction is $76.3 \%$ for one year before bankruptcy, 
$52.6 \%$ for two years before bankruptcy, $44.7 \%$ for three years before bankruptcy. The researchers conclude that the model should be used along with proxies and non-financial models to show the firm's operating environment. Another study of Alareeni and Branson (2012) this study applied the Altman Z-score (1968) model, the sample includes service companies in Jordan, they found that the Altman models could not give early warning for service companies, Another result founded that the Altman models could not give strong indicators to differentiate among failed and non-failed companies. And they recommended that another statistical method must be used within Jordanian companies' context to obtain high accuracy. Other studies such as Gharaibeh and Yacoub (1987), Alomari (2000) and Al-Hroot (2015) developed models using discriminant analysis method, and these studies reached $100 \%$ accuracy rate. A Multidimensional Scaling Approach (MSA) used in two studies; the first one is the study of Alawi and Gharaibeh (2008) while the second study by Jahmani and Dawood (2004) the results varied in prediction accuracy $100 \%$ and $75 \%$ respectively.

We can conclude that studies show differences in the results, while the discriminant analysis shows high predictive ability in many studies, in general researchers in this field reached a satisfactory result. A neural network model has not applied in earlier studies conducted in Jordan, in spite of its success outside Jordan.

After reviewing the related literature and to achieve the objective of the study, the following hypotheses will be tested:

Hypotheses 1: The multilayer perceptron neural network model does not discriminate between bankrupt and non-bankrupt industrial companies in Jordan.

Hypotheses 2: The multilayer perceptron neural network model does not discriminate with a high accuracy between bankrupt and non-bankrupt industrial companies in Jordan.

\section{Research Methodology}

This study is an analytical and applied study of the financial statements of Jordanian industrial sector between 2000 and 2014.

\section{Study Population and Sample}

The study population consisted of the public shareholding Industry Sector in Jordan, that are listed on the Amman stock market, during the period from 2000 to 2015 for a total of 103 companies, of which 25 are bankrupt, 8 companies merged, 2 transformed and the rest are solvent. 


\section{Study Sample}

The sample have been randomly divided into two subsets: the first set for developing or building the model, made up of 14 companies, of which 7 are bankrupt and 7 are non-bankrupt; while the second is a hold-out sample for testing the model, made up of 18 companies, of which 9 are bankrupt and 9 are non-bankrupt. Once we selected the sample, financial ratios are calculated and then entered into the SPSS software to develop the model. Table 1 shows the financial ratios are calculated to be entering into SPSS software to build the model.

Table 1: Financial ratios (Independent variables)

\begin{tabular}{|c|c|c|c|c|c|c|c|c|c|c|}
\hline & $\begin{array}{c}\text { Curren } \\
\text { t ratio }\end{array}$ & $\begin{array}{l}\text { Retu } \\
\text { rn } \\
\text { on } \\
\text { asset } \\
\text { s }\end{array}$ & $\begin{array}{c}\text { Cash } \\
\text { assets } \\
\text { ratio }\end{array}$ & $\begin{array}{l}\text { Debt } \\
\text { ratio }\end{array}$ & $\begin{array}{c}\text { Cash } \\
\text { Flow } \\
\text { Covera } \\
\text { ge } \\
\text { Ratio }\end{array}$ & $\begin{array}{l}\text { Curre } \\
\text { nt } \\
\text { assets } \\
\text { to } \\
\text { total } \\
\text { assets } \\
\text { ratio }\end{array}$ & $\begin{array}{c}\text { Long - } \\
\text { term } \\
\text { debt/t } \\
\text { otal } \\
\text { assets }\end{array}$ & $\begin{array}{c}\text { Mar } \\
\text { gin } \\
\text { Befo } \\
\text { re } \\
\text { Inter } \\
\text { est } \\
\text { and } \\
\text { Tax } \\
\end{array}$ & $\begin{array}{l}\text { Asset } \\
\text { Turn } \\
\text { over } \\
\text { Ratio }\end{array}$ & $\begin{array}{c}\text { Worki } \\
\text { ng } \\
\text { Capit } \\
\text { al } \\
\text { Ratio }\end{array}$ \\
\hline Company name & u1 & $\mathrm{u} 2$ & u3 & $\mathrm{u4}$ & $\mathbf{u} 5$ & u6 & $\mathbf{u} 7$ & $\mathrm{u} 8$ & $\mathbf{u 9}$ & u10 \\
\hline $\begin{array}{l}\text { Jordan Kuwait For } \\
\text { Agriculture \& Food } \\
\text { Products }\end{array}$ & 0.124 & $\begin{array}{c}- \\
0.96 \\
2\end{array}$ & 0.001 & 0.972 & -0.087 & 0.120 & 0.000 & $\begin{array}{c}- \\
0.52 \\
3 \\
\end{array}$ & 0.231 & $\begin{array}{c}- \\
0.852 \\
\end{array}$ \\
\hline $\begin{array}{l}\text { Nayzak Dies \& } \\
\text { Moulds } \\
\text { Manufacturing }\end{array}$ & 0.918 & $\begin{array}{c}- \\
0.06 \\
0\end{array}$ & 0.001 & 0.708 & 0.073 & 0.350 & 0.328 & $\begin{array}{c}- \\
0.22 \\
8\end{array}$ & 0.262 & $\begin{array}{c}- \\
0.031\end{array}$ \\
\hline $\begin{array}{l}\text { Jordan Medical } \\
\text { Corporation }\end{array}$ & 0.200 & $\begin{array}{c}- \\
0.35 \\
2\end{array}$ & 0.109 & 3.113 & -0.043 & 0.621 & 0.007 & $\begin{array}{c}- \\
2.45 \\
2\end{array}$ & 0.144 & $\begin{array}{c}- \\
2.485\end{array}$ \\
\hline $\begin{array}{l}\text { International } \\
\text { Textile } \\
\text { Manufacturing }\end{array}$ & 1.643 & $\begin{array}{c}0.08 \\
1\end{array}$ & 0.011 & 0.452 & -0.027 & 0.366 & 0.230 & $\begin{array}{c}- \\
0.67 \\
7\end{array}$ & 0.060 & 0.143 \\
\hline $\begin{array}{l}\text { United Glass } \\
\text { Industries }\end{array}$ & 33.489 & $\begin{array}{c}0.00 \\
7\end{array}$ & 0.530 & 0.016 & 0.491 & 0.531 & 0.000 & $\begin{array}{c}0.32 \\
5 \\
\end{array}$ & 0.021 & 0.515 \\
\hline $\begin{array}{l}\text { Arab Investment \& } \\
\text { International Trade }\end{array}$ & 1.843 & $\begin{array}{c}- \\
0.06 \\
7\end{array}$ & 0.022 & 0.230 & -0.225 & 0.298 & 0.068 & $\begin{array}{c}- \\
0.21 \\
2\end{array}$ & 0.263 & 0.136 \\
\hline $\begin{array}{l}\text { Arab Food \& } \\
\text { Medical } \\
\text { Appliances }\end{array}$ & 0.132 & $\begin{array}{c}- \\
0.19 \\
8\end{array}$ & 0.002 & 1.043 & -0.136 & 0.138 & 0.000 & $\begin{array}{c}- \\
0.96 \\
4\end{array}$ & 0.096 & $\begin{array}{c}- \\
0.906\end{array}$ \\
\hline $\begin{array}{l}\text { Arab Center For } \\
\text { Pharmaceuticals \& } \\
\text { Chemicals } \\
\text { Industries }\end{array}$ & 14.995 & $\begin{array}{c}0.12 \\
3\end{array}$ & 0.230 & 0.053 & 2.853 & 0.795 & 0.000 & $\begin{array}{c}0.26 \\
6\end{array}$ & 0.497 & 0.742 \\
\hline $\begin{array}{l}\text { Arab Aluminium } \\
\text { Industry }\end{array}$ & 4.043 & $\begin{array}{c}0.08 \\
4\end{array}$ & 0.008 & 0.115 & 1.918 & 0.362 & 0.000 & $\begin{array}{c}0.17 \\
7\end{array}$ & 0.605 & 0.273 \\
\hline $\begin{array}{l}\text { Middle East } \\
\text { Pharmaceutical \& } \\
\text { Chemical } \\
\text { Industries \& } \\
\text { Medical } \\
\text { Appliances } \\
\text { Corporate Actions }\end{array}$ & 4.796 & $\begin{array}{c}0.01 \\
6\end{array}$ & 0.168 & 0.125 & -0.064 & 0.597 & 0.000 & $\begin{array}{c}0.02 \\
0\end{array}$ & 0.834 & 0.473 \\
\hline $\begin{array}{l}\text { Jordan Paper \& } \\
\text { Cardboard }\end{array}$ & 3.609 & $\begin{array}{c}0.06 \\
8\end{array}$ & 0.060 & 0.116 & 0.359 & 0.419 & 0.000 & $\begin{array}{c}0.10 \\
8\end{array}$ & 0.692 & 0.303 \\
\hline
\end{tabular}




\begin{tabular}{|c|c|c|c|c|c|c|c|c|c|c|}
\hline Factories & & & & & & & & & & \\
\hline $\begin{array}{l}\text { Al-ekbal Printing } \\
\text { \& Packaging }\end{array}$ & 3.081 & $\begin{array}{c}0.04 \\
1\end{array}$ & 0.094 & 0.143 & 0.205 & 0.439 & 0.000 & $\begin{array}{c}0.06 \\
9 \\
\end{array}$ & 0.612 & 0.297 \\
\hline $\begin{array}{l}\text { National } \\
\text { Aluminium } \\
\text { Industrial }\end{array}$ & 2.911 & $\begin{array}{c}0.06 \\
5\end{array}$ & 0.089 & 0.255 & 0.423 & 0.422 & 0.432 & $\begin{array}{c}0.16 \\
7\end{array}$ & 0.455 & 0.277 \\
\hline $\begin{array}{l}\text { Universal Modern } \\
\text { Industries }\end{array}$ & 2.346 & $\begin{array}{c}0.03 \\
9\end{array}$ & 0.007 & 0.130 & 0.104 & 0.259 & 0.000 & $\begin{array}{c}0.21 \\
8\end{array}$ & 0.299 & 0.112 \\
\hline
\end{tabular}

\section{Study Procedures and Statistical Processing}

\section{The neural network}

The neural network, also known as: artificial neural network and made up of three groups, or layers, of units: a layer of "input" units is connected to a layer of "hidden" units, which is connected to a layer of "output" units (Stergiou and Siganos, 2016), We chose the neural network to classify bankrupt and non-bankrupt companies on the basis of a set of variables.

\section{Model of Neural Network}

Table 2 shows the case processing summary of the 11 cases originally assigned to the training sample, 3 have been reassigned to the testing sample.

Table 2 Case Processing Summary

\begin{tabular}{|l|c|c|c|}
\hline & & N & Percent \\
\hline Sample & Training & 11 & $78.6 \%$ \\
\hline & Testing & 3 & $21.4 \%$ \\
\hline Valid & & 14 & $100.0 \%$ \\
\hline Excluded & & 0 & \\
\hline Total & & 14 & \\
\hline
\end{tabular}

Table 3 shows the network information. The number of units in the input layer is 10 ratios, the number of hidden layers is one which includes 7 units in this layer using the activation sigmoid function, and the output layer is the status of the company (bankrupt or non-bankrupt).

Table 3 Network information

\begin{tabular}{|l|l|c|c|}
\hline Input Layer & Covariates & 1 & $\mathrm{u} 1$ \\
\hline & & 2 & $\mathrm{u} 2$ \\
\hline & & 3 & $\mathrm{u} 3$ \\
\hline & & 4 & $\mathrm{u} 4$ \\
\hline & & 5 & $\mathrm{u} 5$ \\
\hline & & 6 & $\mathrm{u} 6$ \\
\hline & & 7 & $\mathrm{u} 7$ \\
\hline & & 8 & $\mathrm{u} 8$ \\
\hline & & 9 & $\mathrm{u} 9$ \\
\hline & & 10 & $\mathrm{u} 10$ \\
\hline & Number of Units & & $\mathbf{1 0}$ \\
\hline & Rescaling Method for Covariates & & None \\
\hline
\end{tabular}




\begin{tabular}{|l|l|c|c|} 
Hidden Layer(s) & Number of Hidden Layers & & 1 \\
\hline & Number of Units in Hidden Layer 1 & & 7 \\
\hline & Activation Function & & Sigmoid \\
\hline Output Layer & Dependent Variables & 1 & status \\
\hline & Number of Units & & 1 \\
\hline & Rescaling Method for Scale Dependents & & Normalized \\
\hline & Activation Function & & Sigmoid \\
\hline & Error Function & & Sum of Squares \\
\hline
\end{tabular}

Table 4 show the percentage of incorrect predictions for the model built using neural network is (\%0). And this percentage of incorrect predictions is very low in other words it means that the percentage of correct predictions for this model is very high \%100 this result is supported in table 5 .

Table 4 Model summary

\begin{tabular}{|l|l|l|}
\hline Training & Sum of Squares Error & 0.00 \\
\hline & Relative Error & 0.00 \\
\hline Testing & Sum of Squares Error & 0.00 \\
\hline & Relative Error & 0.00 \\
\hline
\end{tabular}

Table 5 display the cases used to create the model, 7 of the 7 bankruptcy companies previously defaulted are classified correctly. 7 of the 7 Non- bankrupt are classified correctly. Overall, \%100 of the training cases are classified correctly, corresponding with (\% 0$)$ incorrect shown in the model summary table 4 . A better model should correctly identify a higher percentage of the cases.

Table 5: Classification

\begin{tabular}{|l|l|c|c|c|}
\hline \multirow{2}{*}{ Sample } & \multirow{2}{*}{ Observed } & \multicolumn{2}{|c|}{ Predicted } & \multirow{2}{*}{ Percent Correct } \\
\cline { 3 - 5 } & & bankrupt & $\begin{array}{c}\text { Non- } \\
\text { bankrupt }\end{array}$ & \\
\hline Training & bankrupt & 7 & 0 & $100.0 \%$ \\
\hline \multirow{2}{*}{ Testing } & Non- bankrupt & 0 & 7 & $100.0 \%$ \\
\hline & Overall Percent & $50 \%$ & $50 \%$ & $\mathbf{1 0 0 . 0 \%}$ \\
\hline & bankrupt & 7 & 0 & $100.0 \%$ \\
\hline & Non- bankrupt & 0 & 7 & $100.0 \%$ \\
\hline & Overall Percent & $50 \%$ & $50 \%$ & $\mathbf{1 0 0 . 0 \%}$ \\
\hline
\end{tabular}

Table 6 present the synaptic weights (Parameter Estimates) that show the relationship among the input units in a given layer to the units in the following layer (Hidden Layer). The parameter estimates are based on the training sample even if the active dataset is partitioned into training, testing, and holdout data. The number of parameter estimates can become rather large and that these weights are generally not used for interpreting network results. 
Table 6: Parameter Estimates

\begin{tabular}{|c|c|c|c|c|c|c|c|c|c|c|c|c|}
\hline \multirow{3}{*}{$\begin{array}{c}\text { Description } \\
\text { 音导 }\end{array}$} & & \multicolumn{10}{|c|}{ Input Layer } & \multirow{3}{*}{$\begin{array}{c}\text { Output } \\
\text { Layer } \\
\text { x(j) }\end{array}$} \\
\hline & \multirow{2}{*}{ Codes } & $\begin{array}{c}\text { Node } \\
1 \\
\end{array}$ & $\begin{array}{c}\text { Node } \\
2 \\
\end{array}$ & $\begin{array}{c}\text { Node } \\
3 \\
\end{array}$ & $\begin{array}{c}\text { Node } \\
4 \\
\end{array}$ & $\begin{array}{c}\text { Node } \\
5 \\
\end{array}$ & $\begin{array}{c}\text { Node } \\
6 \\
\end{array}$ & $\begin{array}{c}\text { Node } \\
7\end{array}$ & $\begin{array}{c}\text { Node } \\
8\end{array}$ & $\begin{array}{c}\text { Node } \\
9 \\
\end{array}$ & $\begin{array}{c}\text { Node } \\
10 \\
\end{array}$ & \\
\hline & & u1 & u2 & u3 & u4 & u5 & u6 & u7 & u8 & u9 & u10 & \\
\hline \multirow{7}{*}{ 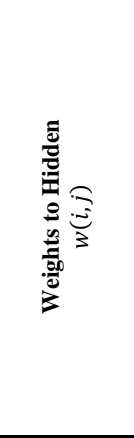 } & $\mathrm{w}(1: 1)$ & 1.048 & 0.254 & $\begin{array}{c}- \\
0.057 \\
\end{array}$ & $\begin{array}{c}- \\
1.805 \\
\end{array}$ & 1.530 & $\begin{array}{c}- \\
0.261 \\
\end{array}$ & $\begin{array}{c}- \\
0.383 \\
\end{array}$ & 1.920 & 0.256 & 1.465 & 2.929 \\
\hline & $\mathrm{w}(1: 2)$ & $\begin{array}{c}- \\
0.212 \\
\end{array}$ & 0.331 & $\begin{array}{c}- \\
0.260 \\
\end{array}$ & $\begin{array}{c}- \\
0.777 \\
\end{array}$ & 1.514 & 0.260 & 0.152 & 1.161 & 0.951 & 0.699 & 3.736 \\
\hline & $w(1: 3)$ & $\begin{array}{c}- \\
1.266 \\
\end{array}$ & $\begin{array}{c}- \\
0.693 \\
\end{array}$ & 0.499 & 1.716 & $\begin{array}{c}- \\
1.645 \\
\end{array}$ & 0.403 & 0.044 & $\begin{array}{c}- \\
2.753 \\
\end{array}$ & 0.209 & $\begin{array}{c}- \\
0.863 \\
\end{array}$ & -5.126 \\
\hline & $\mathrm{w}(1: 4)$ & $\begin{array}{c}- \\
2.565 \\
\end{array}$ & 0.004 & $\begin{array}{c}- \\
0.095 \\
\end{array}$ & 0.414 & $0 . \overline{117}$ & 0.151 & $\begin{array}{c}- \\
0.246 \\
\end{array}$ & 0.025 & 0.214 & $\begin{array}{c}- \\
0.355 \\
\end{array}$ & -2.777 \\
\hline & $w(1: 5)$ & 0.178 & 0.136 & $\begin{array}{c}- \\
0.048 \\
\end{array}$ & $\begin{array}{c}- \\
1.258 \\
\end{array}$ & $\begin{array}{c}- \\
0.950 \\
\end{array}$ & $\begin{array}{c}- \\
0.370 \\
\end{array}$ & $\begin{array}{c}- \\
0.204 \\
\end{array}$ & 0.122 & $\begin{array}{c}- \\
1.106 \\
\end{array}$ & 0.021 & -4.02 \\
\hline & $w(1: 6)$ & $\begin{array}{c}- \\
1.295\end{array}$ & 0.114 & 0.666 & 0.481 & $\begin{array}{c}- \\
0.189 \\
\end{array}$ & 0.356 & $\begin{array}{c}- \\
0.356 \\
\end{array}$ & $\begin{array}{c}- \\
0.632 \\
\end{array}$ & $\begin{array}{c}- \\
0.256 \\
\end{array}$ & $\begin{array}{c}- \\
1.080\end{array}$ & -1.885 \\
\hline & $w(1: 7)$ & 0.955 & 0.261 & $\begin{array}{c}- \\
0.521\end{array}$ & 0.369 & 0.130 & $\begin{array}{c}- \\
0.177\end{array}$ & $\begin{array}{c}- \\
0.423\end{array}$ & 0.442 & 0.545 & $\begin{array}{c}- \\
0.204\end{array}$ & 0.121 \\
\hline
\end{tabular}

There are four steps to get the prediction score:

1- Transforming input nodes to a hidden node $\mathbf{v}(\mathbf{j})$, the hidden nodes $\mathbf{v}(\mathbf{j})$ is given by:

$$
v(j)=\sum_{i=1}^{10} u(i) w(i, j)
$$

The lower part of Table 7 shows the results of the application of the previous equation No. 1. However, these values v(j) are not finally because they are not actual values that algorithm uses.

Table .7 hidden node v(j)

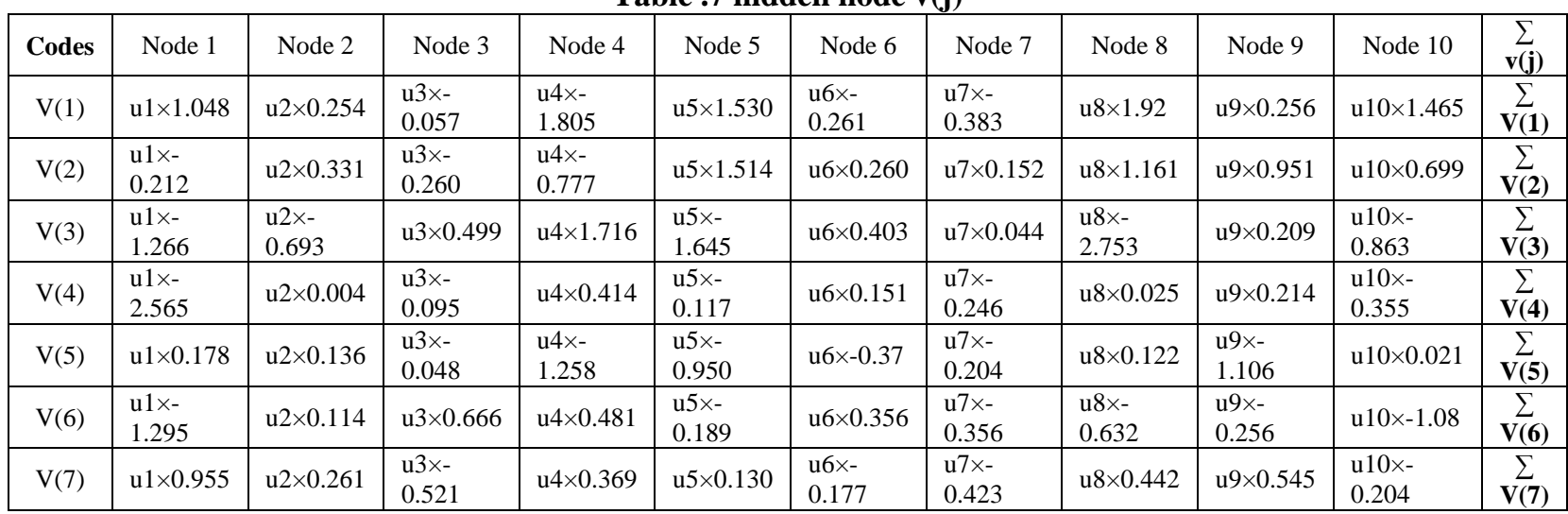

2- Transforming values to actual values

The values $\sum \mathrm{v}(\mathrm{j})$ must transformed to so-called "thresholded" value to be actual values, the thresholded values fall between 0 and 1 (Gosavi, 2015), these hidden node $\sum \mathrm{v}(\mathrm{j})$ will be transformed using the sigmoid function, is given by : 


$$
h(j)=\frac{1}{1+e^{-v(j)}}
$$

No. 2.

Table 8 shows the results of the application of the previous equation

Table .8 Transforming hidden node $\sum v(j)$

\begin{tabular}{|c|c|}
\hline$\sum \mathbf{V}(\mathbf{j})$ & $\boldsymbol{h}(\boldsymbol{j})$ \\
\hline$\sum \mathbf{V}(\mathbf{1})$ & $1 /\left(1+e^{-v(1)}\right)$ \\
\hline$\sum \mathbf{V}(\mathbf{2})$ & $1 /\left(1+e^{-v(2)}\right)$ \\
\hline$\sum \mathbf{V}(3)$ & $1 /\left(1+e^{-v(3)}\right)$ \\
\hline$\sum \mathbf{V}(\mathbf{4})$ & $1 /\left(1+e^{-v(4)}\right)$ \\
\hline$\sum \mathbf{V}(5)$ & $1 /\left(1+e^{-v(5)}\right)$ \\
\hline$\sum \mathbf{V}(\mathbf{6})$ & $1 /\left(1+e^{-v(6)}\right)$ \\
\hline$\sum \mathbf{V}(7)$ & $1 /\left(1+e^{-v(7)}\right)$ \\
\hline
\end{tabular}

3- Transforming the links between the hidden nodes and output node

The weights on the link from hidden node to the output node is $x(j)$. then the output node's value can be calculated using the below formula :

$$
o(j)=\sum_{i=1}^{10} h(j) x(j)
$$

Table 9 shows the results of the application of the previous equation No. 3.

Table .9 calculating the output node o $(j)$

\begin{tabular}{|c|c|c|}
\hline $\boldsymbol{h}(\boldsymbol{j})$ & $\boldsymbol{x}(\boldsymbol{j})$ & $\boldsymbol{o}(\boldsymbol{j})$ \\
\hline $1 /\left(1+e^{-v(1)}\right)$ & 2.929 & $\mathrm{~h}(1) \times \mathrm{x}(1)$ \\
\hline $1 /\left(1+e^{-v(2)}\right)$ & 3.736 & $\mathrm{~h}(2) \times \times(2)$ \\
\hline $1 /\left(1+e^{-v(3)}\right)$ & -5.126 & $\mathrm{~h}(3) \times \times(3)$ \\
\hline $1 /\left(1+e^{-v(4)}\right)$ & -2.777 & $\mathrm{~h}(4 \times \times(4)$ \\
\hline $1 /\left(1+e^{-v(5)}\right)$ & -4.020 & $\mathrm{~h}(5) \times \times(5)$ \\
\hline $1 /\left(1+e^{-v(6)}\right)$ & -1.885 & $\mathrm{~h}(6) \times \times(6)$ \\
\hline $1 /\left(1+e^{-v(7)}\right)$ & 0.121 & $\mathrm{~h}(7) \times \times(7)$ \\
\hline \multicolumn{2}{|c|}{ Total $\left(\sum_{i=1}^{10} h(j) x(j)\right)$} & Value * \\
\hline
\end{tabular}

* The value vary from company to other related to the financial ratios

4- In the last step we have to transform the value o(j) similar to calculation in step 2 using the sigmoid function, is given by :

$$
\text { predection score }(p s)=\frac{1}{1+e^{-v(j)}}
$$

Tables 10 show the results after testing the model and multilayer perceptron neural network was used. The model comprises an input layer of ten neurons for ten financial ratios, a hidden layer of seven hidden nodes, and output layer of one node. The output values vary between zero and one; using a cut-off level of 0.5 to classify the output values into two classes' bankrupt and non-bankrupt companies, output value of less than 0.5 was 
classified as a bankrupt company, while one with an output value of greater than or equal to 0.5 was classified as a non-bankrupt company.

On the hold-out test data, using a cut-off level of 0.5 in the output to signify a bankrupt versus a non-bankrupt, the trained network correctly classified all 18 test cases, comprising 9 bankruptcy companies and 9 nonbankruptcy companies. That is, the network's accuracy rate is $100 \%$ for bankrupt, non-bankrupt, and overall, in each case. The results of neural network model are summarized in Table 10 for the cases used to build the model. While the hold-out sample results are presented in Table 11.

Table 10: Testing the model

\begin{tabular}{|c|l|c|c|c|}
\hline Serial & \multicolumn{1}{|c|}{ Company Name } & Prediction Score & Classification & Actual Status \\
\hline 1 & $\begin{array}{l}\text { Jordan Kuwait For Agriculture \& } \\
\text { Food Products }\end{array}$ & 0.001 & Distressed & Distressed \\
\hline 2 & $\begin{array}{l}\text { Nayzak Dies \& Moulds } \\
\text { Manufacturing }\end{array}$ & 0.236 & Distressed & Distressed \\
\hline 3 & Jordan Medical Corporation & 0.000 & Distressed & Distressed \\
\hline 4 & International Textile Manufacturing & 0.491 & Distressed & Distressed \\
\hline 5 & United Glass Industries & 0.288 & Distressed & Distressed \\
\hline 6 & $\begin{array}{l}\text { Arab Investment \& International } \\
\text { Trade }\end{array}$ & 0.128 & Distressed & Distressed \\
\hline 7 & Arab Food \& Medical Appliances & 0.001 & Distressed & Distressed \\
\hline 8 & $\begin{array}{l}\text { Arab Center For Pharmaceuticals \& } \\
\text { Chemicals Industries }\end{array}$ & 0.999 & non distress & non-distress \\
\hline 9 & Arab Aluminium Industry & 0.999 & non distress & non-distress \\
\hline & $\begin{array}{l}\text { Middle East Pharmaceutical \& } \\
\text { Chemical Industries \& Medical }\end{array}$ & 0.707 & non distress & non-distress \\
\hline 10 & Appliances Corporate Actions & 0.982 & non distress & non-distress \\
\hline 11 & Jordan Paper \& Cardboard Factories & 0.956 & non distress & non-distress \\
\hline 12 & Al-ekbal Printing \& Packaging & non distress & non-distress \\
\hline 13 & National Aluminium Industrial & 0.990 & non distress & non-distress \\
\hline 14 & Universal Modern Industries & 0.913 & & \\
\hline
\end{tabular}

Table 11: hold-out sample testing

\begin{tabular}{|c|l|c|c|l|}
\hline Serial & \multicolumn{1}{|c|}{ Company Name } & Prediction Score & Classification & Actual Status \\
\hline 1 & Jordan Ceramic Industries & 0.241 & Distressed & Distressed \\
\hline 2 & Jordan Tanning & 0.178 & Distressed & Distressed \\
\hline 3 & National Industries & 0.333 & Distressed & Distressed \\
\hline 4 & Rafia Industrial & 0.214 & Distressed & Distressed \\
\hline 5 & Jordan Spinning \& Weaving & 0.297 & Distressed & Distressed \\
\hline 6 & Kawther Investments & 0.072 & Distressed & Distressed \\
\hline 7 & Arab Engineering Industries & 0.411 & Distressed & Distressed \\
\hline 8 & $\begin{array}{l}\text { National Textile \& Plastics } \\
\text { Industries }\end{array}$ & 0.319 & Distressed & Distressed \\
\hline 9 & International Silica Industries & 0.838 & Non-distress & Non-distress \\
\hline 10 & $\begin{array}{l}\text { Alkindi Pharmaceutical } \\
\text { Industries }\end{array}$ & 0.732 & Non-distress & Non-distress \\
\hline 11 & Travertine & 0.892 & Non-distress & Non-distress \\
\hline 12 & The Jordanian Pharmaceutical & 0.976 & Non-distress & Non-distress \\
\hline 13 & Mational Steel Industry & 0.929 & Non-distress & Non-distress \\
\hline
\end{tabular}




\begin{tabular}{|c|l|c|c|l|}
\hline 14 & $\begin{array}{l}\text { Dar Al Dawa Development \& } \\
\text { Investment }\end{array}$ & 0.987 & Non-distress & Non-distress \\
\hline 15 & The Jordan Worsted Mills & 0.881 & Non-distress & Non-distress \\
\hline 16 & Jordan Paper \& Cardboard & 0.999 & Non-distress & Non-distress \\
\hline 17 & Factories & 0.984 & Non-distress & Non-distress \\
\hline 18 & Gendan Dairy & 0.996 & Non-distress & Non-distress \\
\hline
\end{tabular}

\section{Conclusion:}

Considering the results neural network have shown high predictive ability in classifying the Jordanian companies status from financial ratios. A sample of 7 bankrupt company and 7 matched non-bankrupt company for the period 2000-2015.

In this study, the multilayer perceptron neural network (MPNN) was used. The model comprises an input layer of ten neurons for ten financial ratios, a hidden layer of seven hidden nodes, and output layer of one node. The output values vary between zero and one; using a cut-off level of 0.5 to classify the output values into two classes bankruptcy versus a nonbankruptcy, output value of less than 0.5 was classified as a bankrupt company, while one with an output value of greater than or equal to 0.5 was classified as a non-bankrupt company.

The model can predicts bankruptcy of Jordanian industrial listed companies with the accuracy of $100 \%$ for 1 year before. On the hold-out sample, the network correctly classified all 18 test cases, comprising 9 bankrupt versus 9 non-bankrupts. The results are associated with the findings of Raghupathi \& Schkade and Raju (1991), Odom \& Sharda (1990) and Koh \& Tan (1999). They found that neural network algorithms can be investigated further as potential models for bankruptcy prediction and achieve the best overall prediction results.

For further research in Jordan other bankruptcy prediction models can be applied and compare the results and also application of Radial basis function network (RBFN) models is suggested for future research.

\section{References:}

Alareeni, B. A., \& Branson, J. (2012). Predicting listed companies’ failure in Jordan using Altman models: A case study. International Journal of Business and Management, 8(1), p113.

Al-hroot, Y. (2015), The influence of sample size and selection of financial ratios in bankruptcy model accuracy Economic Review: Journal of Economics and Business, 2015; XIII (1)7-19.

Alkhatib, K., \& Al Bzour, A. E. (2011). Predicting corporate bankruptcy of Jordanian listed companies: Using Altman and Kida models. International Journal of Business and Management, 6(3), p208. 
FitzPatrick, Paul J., Ph.D. 'A Comparison of the Ratios of Successful Industrial Enterprises With Those of Failed Companies'. The Certified Public Accountant Beaver 1968. Journal of ccounting Research. (In three issues: October, 1932, p. 598-605; November, 1932, p. 656-662; December, 1932, p. 727-731)

Gosavi, A. (2015). Simulation-Based Optimization: An Overview. In Simulation-Based Optimization (pp. 29-35). Springer US.

Goss, E. P., \& Ramchandani, H. (1995). Comparing classification accuracy of neural networks, binary logit regression and discriminant analysis for insolvency prediction of life insurers. Journal of Economics and Finance, 19(3), 1-18.

Karamzadeh, M. S. (2013). Application and Comparison of Altman and Ohlson Models to Predict Bankruptcy of Companies. Research Journal of Applied Sciences, Engineering and Technology, 5(6), 2007-2011.

Koh, H. C., \& Tan, S. S. (1999). A neural network approach to the prediction of going concern status. Accounting and Business Research, 29(3), 211-216. Mehrazin, Alireza, et al. "Radial Basis Function in Artificial Neural Network for Prediction of Bankruptcy." International Business Research 6.8 (2013): p121.

Odom, M. D., \& Sharda, R. (1990). A neural network model for bankruptcy prediction. In 1990 IJCNN International Joint Conference on neural networks (pp. 163-168).

Ohlson, J. A. (1980). Financial ratios and the probabilistic prediction of bankruptcy. Journal of accounting research, 109-131.

Raghupathi, W., Schkade, L. L., \& Raju, B. S. (1991, January). A neural network application for bankruptcy prediction. In System Sciences, 1991. Proceedings of the Twenty-Fourth Annual Hawaii International Conference on (Vol. 4, pp. 147-155). IEEE.

Stergiou, C., \& Siganos, D. 1. (2016). Introduction to Neural Networks 1.1 What is a neural network? 1.2 Historical background 1.3 Why use neural networks? 1.4 Neural networks versus conventional computers-a comparison. Available on

http://www.doc.ic.ac.uk/ nd/surprise_96/journal/vol4/cs11/report.html 